\title{
Geometry Investigation and Performance Optimization of a Single-Mass Piezoelectric 6-DOF IMU
}

\author{
Hela Almabrouk \\ Université de Sfax, Ecole Nationale d'Ingénieurs de Sfax, Sfax, Tunisia \\ Faculté des Sciences de Monastir, Université de Monastir, Monastir, Tunisia \\ GeePs Laboratory, Université Paris Sud, Gif sur Yvette, France
}

\author{
Brahim Mezghani \\ Université de Sfax, Ecole Nationale d'Ingénieurs de Sfax \\ UR-METS, Sfax, Tunisia
}

\author{
Guillaume Agnus \\ Université Paris Sud, C2N - UMR 9001 CNRS \\ Palaiseau, France \\ Yves Bernard \\ GeePs Laboratory, Université Paris Sud \\ Gif sur Yvette, France
}

\author{
Sinda Kaziz \\ Université de Sfax, Ecole Nationale d'Ingénieurs de Sfax, Sfax, Tunisia \\ Faculté des Sciences de Monastir, Université de Monastir, Monastir, Tunisia \\ Fares Tounsi \\ Université de Sfax, Ecole Nationale d'Ingénieurs de Sfax, \\ UR-METS, Sfax, Tunisia
}

\begin{abstract}
This paper explores the fundamental steps towards the development of a 6-axis piezoelectric Inertial Measurement Unit (IMU). The main specification of the reported device is its ability to concurrently detect 3 -axis acceleration and angular velocity using a single mass-based design. This work represents a detailed numerical analysis based on a finite element model. Experimental reported data are exploited to validate the FEM model in terms of acceleration detection which is ensured through the direct piezoelectric effect. The angular rate is detected thanks to the Coriolis effect by ensuring drive and sense modes. Using a Finite Element Analysis (FEA), light was shed on the different basic parameters that influence the sensor performance in order to present an optimized design. A detailed geometrical investigation of factors such as anchor position, optimized locations for sensing electrodes, proof-mass dimensions, PZT thickness, and operating frequency is illustrated. The 6-DOF sensor outputs are extracted in terms of the original and the optimized design. The amelioration rate of sensitivity is found to be up to $165 \%$ for linear acceleration, while for angular rate sensing, the lateral sensitivity is ameliorated by about $330 \%$ and is multiplied by around ten times in the normal axis. The optimized design exhibits a good acceleration sensitivity of $260 \mathrm{mV} / \mathrm{g}$ in the lateral axis and $60.7 \mathrm{mV} / \mathrm{g}$ in the $\mathrm{z}$-axis. For angular rate sensing, the new design is more sensitive along the longitudinal axis than the lateral one. Sensitivity values are found to be $2.65 \mu \mathrm{V} / \mathrm{rad} / \mathrm{s}$ for both $\mathrm{x}$-and $\mathrm{y}$-axis, and $1.24 \mathrm{~V} / \mathrm{rad} / \mathrm{s}$ for the $\mathrm{z}$-axis.
\end{abstract}

Keywords-acceleration; Coriolis effect; angular rate detection; FEM simulations; inertial measurement unit; piezoelectric detection

\section{INTRODUCTION}

The development of multi-axis inertial sensors has been rising considerably. The scientific community has shed light on multi-motion sensing exemplified in Inertial Measurement
Units (IMUs). Such systems are employed to measure linear and rotational motion data such as gravitational forces and angular rates. Nowadays, IMU systems are becoming important and are largely investigated [1-4] due to the fact that they are among the primary used systems in various fields, e.g. they are the main means of environment interaction for robotic applications and their system development $[5,6]$. Also, IMU devices have shown great contribution in medical rehabilitation fields $[7,8]$, they have been broadly investigated in industrial quality control systems [9], navigation systems [10, 11], motion sensing and portable positioning systems $[12,13]$, and in smart sensors for Internet of Things (IoT) applications [14, 15]. Multiple-axis sensing systems have been developed relying on several physical effects, most known for motion capture are capacitive, thermal, piezoelectric and piezoresistive. Nevertheless, piezoelectric materials have presented great performance in many inertial sensors. Especially, the piezoelectric effect exhibits many advantages compared to other sensing mechanisms such as low power consumption, wide dynamic range frequency, and compatibility with reduced device dimensions [16, 17]. Furthermore, their self-powering feature is the most attractive advantage of piezoelectric sensors. Indeed, the piezoelectric effect is manifested by a transfer of mechanical energy into electrical and vice versa, i.e. the direct effect is triggered by a deformation that will generate a mechanical stress which in turn produces an electrical voltage. Similarly, the converse effect is illustrated when an electric field input induces a mechanical deformation. For IMUs, the number of Degrees Of Freedom (DOF) reflects the number of axes that the system is sensitive to. Therefore, a 6-DOF motion sensing application is incorporating $x$-, $y$-, and $z$-axis measurement of all possible accelerations and 3 -axis angular rate components. Several 3-

Corresponding author: Hela Almabrouk (hela.almabrouk@enis.rnu.tn) 
DOF or multi-axis sensors for accelerometers and for gyroscopes have been reported [18-22]. These devices can be co-integrated into a single system to form a 6-axis IMU. However, their size and cost, composed by an assembly of two or more separate sensors, can be annoying for certain applications. In addition, each multi-axis accelerometer or gyro sensor requires generally separate drive and sense electronics, leading to an increase in cost and complexity of the designed inertial unit. Therefore, 6 -axis detection for linear and angular motion sensors were proposed using piezoresistive [23], and piezoelectric principle sensing [24-25] based on a single inertial mass. In exploiting the Coriolis effect with piezoelectric sensors, a sole work has been carried out to cointegrate these two different inertial measurement types into one device through the same detecting elements [25].

In the current study, an in-depth analysis of a single-mass piezoelectric IMU structure to be used as a 6-axis inertial sensor was carried out. The output of this Sensor Under Development (SUD) is proportional to each of the 3-axis angular rate components in addition to the 3 -axis linear accelerations. The structure of this SUD is inspired from the one presented in $[25,26]$. Using the reported experimental values, a newly developed FEM model, using COMSOL Multiphysics software, has been performed and validated. This 3D FEM model was then used for the performance analysis of the SUD behavior in both drive and sense modes. The novelty of this work is mainly manifested through the investigation of key parameters that influence the performances of the reported structure and their analysis. The main aim of this study is to evaluate a 6-DOF piezoelectric detection system and enhance its sensing performance through the optimization of its key geometrical parameters.

\section{IMU DESIGN PRESENTATION}

The SUD structure is able to detect 3-axis accelerations and 3-axis angular velocities using a single-mass design. The analyzed design, based on the scheme reported in [25], uses nine electrodes implemented on the top of a piezoelectric membrane for both drive and sense modes as presented in Figure 1. Sense electrodes, placed on the inner and outer rings, are used to detect induced stresses and are denoted $\mathrm{S}_{\mathrm{x} 1}, \mathrm{~S}_{\mathrm{x} 2}, \mathrm{~S}_{\mathrm{y} 1}$, $\mathrm{S}_{\mathrm{y} 2}$, and Ring. Drive electrodes, located on the middle ring, are used to actuate the IMU design and are denoted as $\mathrm{D}_{\mathrm{x} 1}, \mathrm{D}_{\mathrm{x} 2}$, $\mathrm{D}_{\mathrm{y} 1}$, and $\mathrm{D}_{\mathrm{y} 2}$. A circular bottom conductive membrane, made of high precision Elinvar (nickel-iron-chromium alloy), is used as a potential reference and a supporting substrate (anchor). A proof-mass is attached at the bottom center of the Elinvar thick membrane and used as an oscillator. The linear acceleration detection is based on the use of the direct piezoelectric effect. Hence, an incident acceleration motion on the seismic mass induces strain in the piezoelectric membrane and the generated voltage is collected by the corresponding sensing electrodes placed on the top piezoelectric membrane. For angular rate detection, the Coriolis Effect is exploited. This phenomenon is designated when a moving object is subjected to an angular motion in a rotating frame. Practically, the act of the applied rotation on the oscillated proof-mass gives rise to a new fluctuation component that is produced by the Coriolis Effect.

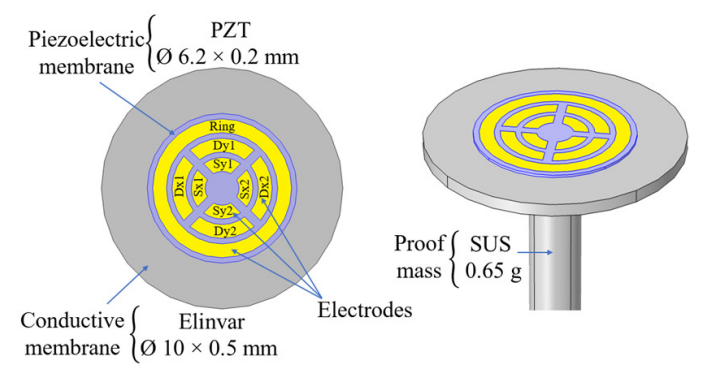

Fig. 1. Design presentation of the piezoelectric 6-DOF IMU.

\section{FEM MODEL VALIDATION}

No dimensions' details were given in [25] concerning the proof-mass. Only the used material, stainless steel (or SUS), and its weight, $0.65 \mathrm{~g}$ were declared. Thus, the length and radius of the seismic mass will be determined based on a comparison study between our FEM simulations and the experimental values specified in [25]. FEM simulations have been extended for an acceleration range of 1 to $10 \mathrm{~g}$. In response to $10 \mathrm{~g}$ of $x$ and $z$-axis linear accelerations, distributions of output voltages on the membrane are shown in Figures 2(a)-(b). For normal acceleration input, the output voltage is collected from the sum of all four sense electrodes (Figure 2(a)). For longitudinal accelerations, the voltage is collected from the potential difference between the two electrodes located in the direction of acceleration (Figure 2(b)). The corresponding results of output voltages are plotted in Figure 3. This good agreement between simulation and the reported experimental results is obtained for radius $R=1 \mathrm{~mm}$ and length $L=26.52 \mathrm{~mm}$.

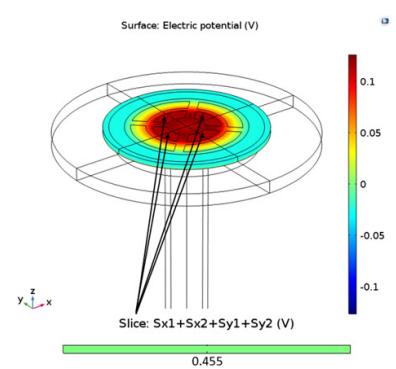

(a)

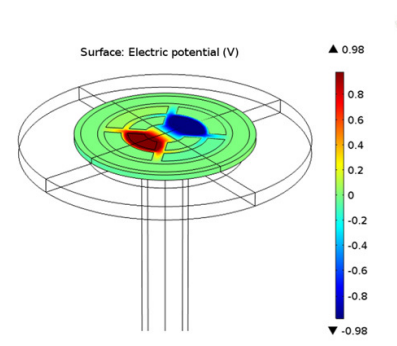

(b)
Fig. 2. Distribution of the output voltages on top of the IMU membrane using $R=1 \mathrm{~mm}$ and $L=25.83 \mathrm{~mm}$ in response to a $10 \mathrm{~g}$ acceleration along the (a) $x$-axis and (b) $z$-axis.

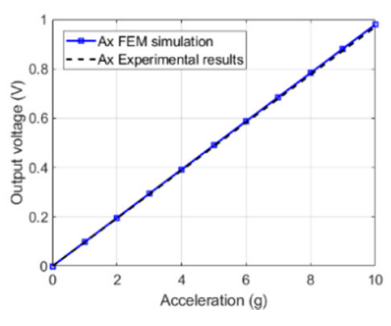

(a)

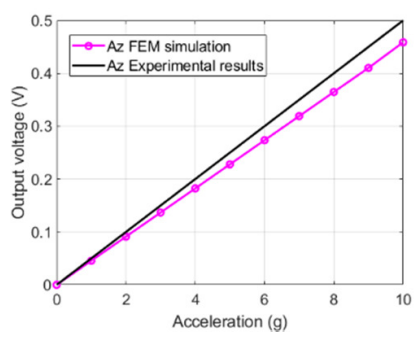

(b)
Fig. 3. FEM simulation values and experimental results from [25] of the IMU using $R=1 \mathrm{~mm}$ and $L=25.83 \mathrm{~mm}$ in response to accelerations along (a) $\mathrm{x}$-axis and (b) z-axis. 


\section{GEOMETRY OPTIMIZATION}

In this section, an in-depth analysis of the structural system's behavior in response to different design factors is introduced. FEM simulations of the basic structure design are investigated in order to highlight the geometrical parameters that influence the system's performance. The structure optimization is based on the specific location of the anchor position, sense and drive electrode locations and proof-mass dimensions, PZT thickness, and optimum operating frequency. Thus, our contribution is to enrich the characterized sensor with an FEA study in order to optimize the design.

\section{A. Position of Anchor and Sense Electrodes}

The anchor design and the electrode positions are important design parameters. Three different positions are inspected to explore and then determine the most efficient anchor position in order to localize the induced optimum stress. As illustrated in Figure 4(a), position 1 consists in fixing the middle bottom surface between the Elinvar and the PZT cylinders. In position 2 , which is the original anchor position, the bottom of the Elinvar cylinder is fixed up to the limit of the PZT cylinder and position 3 consists in fixing the bottom of the Elinvar cylinder beyond the limit of the PZT element.

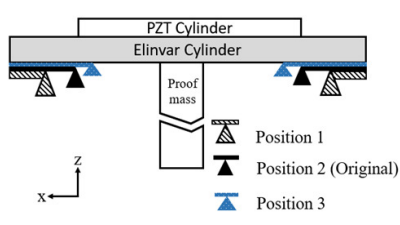

(a)

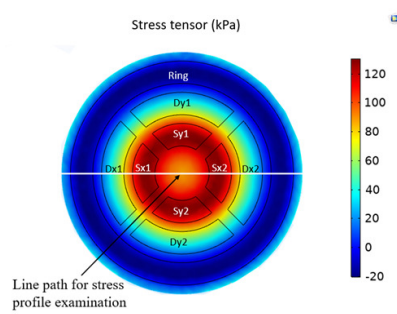

(b)
Fig. 4. (a) Illustration of the three possible IMU structure anchoring cases, (b) $2 \mathrm{D}$ stress profile on the top piezoelectric membrane under a $10 \mathrm{~g} z$-axis acceleration for the 3 case position.

For each of the three anchor positions given in Figure 4(a), $x$ - and $z$-axis excitation accelerations are applied on the IMU structure through FEM simulations, and lateral stress on the PZT cylinder top surface is inspected. This provides preliminary valuable information that help choosing the optimal anchor setting between the three examined ones. In Figure $4(\mathrm{~b})$, the generated stress distribution is shown using position 3 when the IMU is submitted to $10 \mathrm{~g}$ acceleration along the $z$-axis. Stress distribution along the PZT diameter when the IMU is submitted to $x$ - and $z$-axis accelerations are plotted in Figure 5(a) and Figure 5(b) respectively. We note that the stress is opposite and symmetrically distributed along the piezoelectric membrane with respect to its center. For the $x$ axis force, the stress values produced with position 2 and position 3 are close. Nevertheless, our choice is pointed to position 3 since it is the only position that provides optimum stress in ring location which is useful for $z$-axis detection. It is worth noting that the structure is less sensitive to vertical excitations, and especially at the external ring, which could be explained by the fact that the seismic effect of the mass is reduced during a vertical excitation due to the high normal stiffness of the IMU structure. Consequently, priority will be given to placing the Ring in the zone of maximum stress.

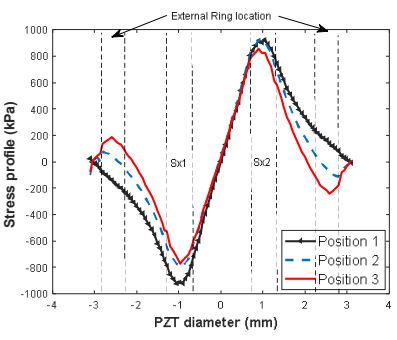

(a)

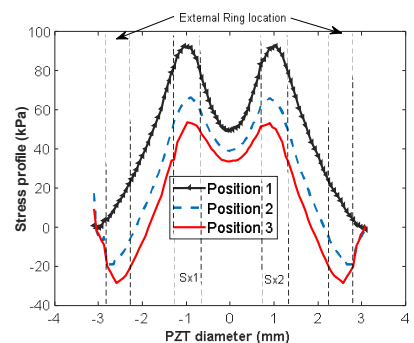

(b)
Fig. 5. Illustration of $1 \mathrm{D}$ stress profile relative to positions 1 to 3 of the anchor when the IMU is under (a) $x$-axis and (b) $z$-axis acceleration.

By analyzing Figure 5, we can conclude that all sense electrodes (i.e. $\mathrm{S}_{\mathrm{x} 1}, \mathrm{~S}_{\mathrm{x} 2}, \mathrm{~S}_{\mathrm{y} 1}, \mathrm{~S}_{\mathrm{y} 2}$ and Ring) should be localized in such a way that their median lines coincide with the stress peak. This will ensure that they will cover the distributed stressed area as depicted in Figure 4(b). The electrodes' width has been set to $575 \mu \mathrm{m}$ with a spacing of $125 \mu \mathrm{m}$. These dimensions allow placing all electrodes properly in the available area where the stress is maximum.

\section{B. Proof-Mass Length vs. Mechanical Resonance Frequency}

The seismic mass is considered as a key performance element since it is used as an oscillator for drive mode generation and motions are transmitted to the piezoelectric membrane only through the proof-mass causing its deflection. Hence, proof-mass dimensions should be carefully optimized for best performance. The cylindrical proof-mass length $L$ is related to its weight, $m$, radius $R$, and material density, $\rho=7800 \mathrm{~kg} / \mathrm{m}^{3}$ by:

$$
L=\frac{m}{\rho \pi R^{2}}
$$

For maximum proof-mass oscillation, the frequency of the Drive signal used for excitation should be set around the mass resonance frequency, which is closely related to the mass length. Therefore, the effect of the proof-mass length on the natural frequency of the structure has been examined. On the other hand, it is essential to examine the proof-mass tip flexibility along the $x$ - and $z$-directions to find which direction is more sensitive. Hence, the SUD design is simulated under $x$ and $z$-axis acceleration excitation motion $(10 \mathrm{~g})$ as a function of the proof-mass length. In Figure 6, natural frequency and displacement magnitude are plotted, in both $x$-and $z$-directions, of the seismic mass middle tip for different mass lengths. A point worth emphasizing is that displacement and frequency are inversely proportional, i.e. when the mass is longer, displacement increases, but natural frequency decreases. Moreover, it should be noted that the structure is more sensitive along the $x$-axis than the $z$-axis since $x$-axis displacement is much more pronounced. This would certainly influence the sensor outputs versus lateral and longitudinal excitations.

\section{PZT Thickness Influence}

Piezoelectric properties and device sensitivity can be dependent on several factors, one of them being the PZT 
thickness $[29,30]$. In this context, we exhibit the effect of the PZT film thicknesses, ranging from 200 to $400 \mu \mathrm{m}$ on the produced tensor stress under an applied static acceleration force to the SUD design. The results are exhibited in Figure 7.

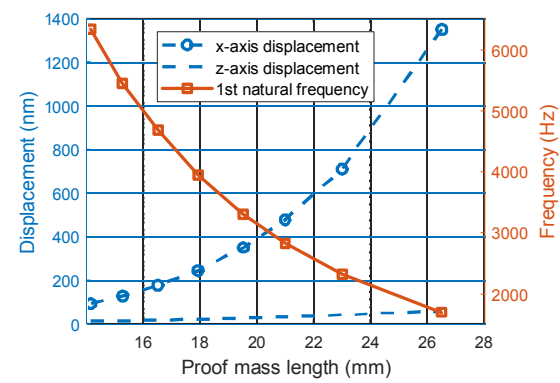

Fig. 6. Tilt displacement of the proof-mass middle tip and structure resonance frequency versus length of the proof-mass under $10 \mathrm{~g}$ accelerations.

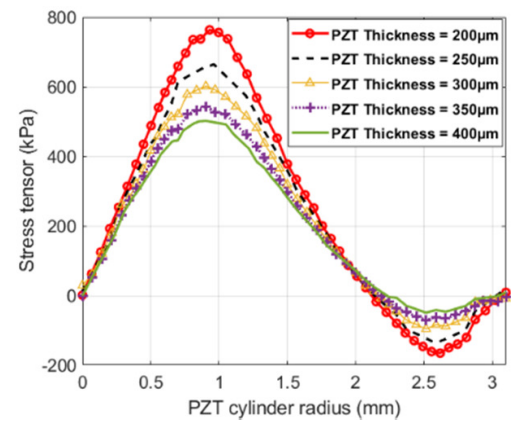

Fig. 7. Stress profile in response to different PZT thickness under static acceleration input.

It has been underlined that the stress tensor is directly dependent on the PZT film thickness. The optimal film thickness for maximum mass-sensitivity of the design is in the range of $200 \mu \mathrm{m}$. However, two points worth noting must be considered: firstly, the optimum PZT thickness must be chosen not only in terms of highest stress values but also in terms of respect to the maximum stress allowed by the material in order to avoid structural damages during system operation. In fact, the maximum allowable stress is commonly evaluated at 50MPa for soft PZT ceramics [28]. Secondly, we have to pay attention to the additional stress generation by the mass oscillation. Thus, stress values are considered in response to linear and angular motion under drive mode and it has been found that with $200 \mu \mathrm{m}$ of PZT thickness, a safety factor of 12 and 3 is ensured for linear and angular motion sensing respectively. These values are listed in Table I. Therefore, with this optimal thickness, IMU outputs can be significantly ameliorated with no mechanical damages.

TABLE I. STRESS TENSOR OF THE SUD DESIGN $(200 \mu \mathrm{m}$ PZT THICKNESS) IN RESPONSE TO MAXIMUM LOAD VALUES UNDER DRIVE MODE

\begin{tabular}{|c|c|c|}
\hline Load (max) & Stress tensor & Safety factor \\
\hline Acceleration $10 \mathrm{~g}$ & $4 \mathrm{MPa}$ & 12 \\
\hline Angular rate $2000 \mathrm{rad} / \mathrm{s}$ & $14 \mathrm{MPa}$ & 3 \\
\hline
\end{tabular}

\section{Frequency Response}

The operating frequency is very important since it defines the drive mode frequency which is the basic part of the angular rate detecting system. This mode must be accurately set to ensure a proof-mass oscillation in the $x / y$ plan for 3 -axis angular rate sensing. The choice of the drive signal frequency is preliminarily extracted from an eigen frequency study of the SUD structure showing its behavior under each mode (Figure 8). This study shows that the first resonant frequency mode is manifested by two symmetrical modes (mode $1 \mathrm{a}$ and mode $1 \mathrm{~b}$ ) showing deformations along $x$ - and $y$-axis respectively which corresponds well to our desired case. Furthermore, the dynamic system response of the SUD is analyzed under the acceleration motion input in order to assure that the chosen frequency is suited for both linear and angular motion sensing. The dynamic response is based on (2) governing a second order system of the acceleration sensing model [27] (mass-spring damper system):

$$
m \ddot{x}+c \dot{x}+k x=-m a=-F
$$

where $m$ is the mass of the proof-mass, $a$ is the acceleration experienced to the proof-mass, $x$ is the corresponding displacement, $c$ is the damping coefficient, and $k$ is the spring constant. By taking the Laplace transform of this equation, the dynamic response is depicted in (3):

$$
X(s)=\frac{F(s)}{m s^{2}+c s+k}
$$

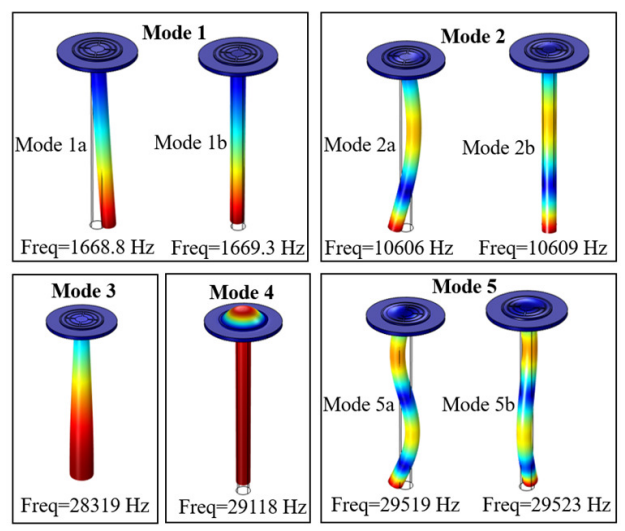

Fig. 8. Modal analysis of the analyzed IMU structure.

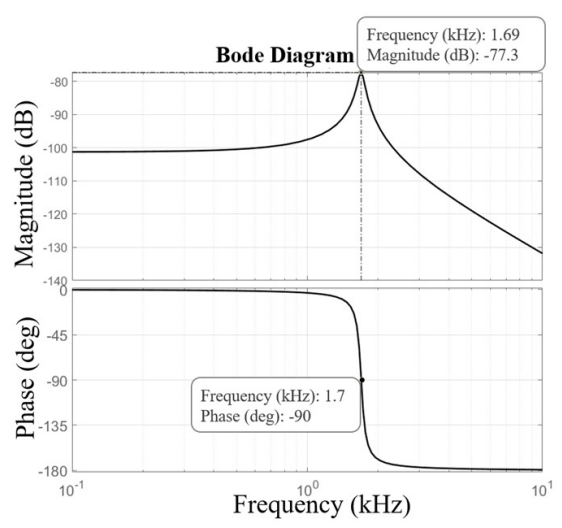

Fig. 9. Bode plot of the dynamic response of the SUD. 
The transfer function is numerically examined and the magnitude (in $\mathrm{dB}$ ) and phase (in degrees) of the system response are depicted in Figure 9. Through this illustration, the first eigen frequency mode is manifested and the bandwidth of the SUD is found to be $110 \mathrm{~Hz}$. Accordingly, the excitation frequency to be chosen is in the vicinity of the oscillation mode 1. Indeed, exciting in the vicinity of the first resonant frequency avoids the appearance of nodes during the oscillation, and it permits consequently full exploitation of the seismic effect of the proof-mass.

\section{SIX-DOF DETECTION EVALUATION}

Taking in account all the optimized parameters, the 6-DOF outputs of the new design have been investigated. FEM simulations were performed on the oscillating design to estimate acceleration and angular velocity responses retrieved directly from the detection electrodes, i.e. before being processed by the conditioning electronic blocks. Applied accelerations are converted into a mechanical force given by Newton's second law. The movements of the seismic mass are firstly transformed into a corresponding displacement on the piezoelectric surface, and then into an output voltage produced on sense electrodes thanks to the direct piezoelectric effect. In the presence of a rotational motion, a Coriolis force $F_{c}$ is generated and acts always perpendicularly to the directions of angular rate to be measured and the proof-mass oscillation. Thus, the new displacement amplitude raised by the Coriolis effect is proportional to the angular rate to be measured using the direct piezoelectric effect. The efficiency of the piezoelectric effect, used for both excitation and detection modes, contributes significantly to the determination of the IMU performance. The Coriolis force is given by:

$$
\overrightarrow{F_{c}}=2 m_{o s c i} \vec{v} \times \vec{\Omega}
$$

where $v$ is the applied drive velocity component on the oscillator having a mass $m_{\text {osci }}$ and $\Omega$ is the angular rate to be measured. The Coriolis force expression depends directly on the drive velocity of the proof-mass which represents the derivative of its displacement. Hence, in order to improve the generated Coriolis force, proof-mass displacement should be maximized.

\section{A. Drive Mode Setting}

Drive mode must be configured in such a manner that an oscillation motion of the proof-mass in the $x / y$-plane is ensured. This is obtained through the application of four different AC signals that are phase shifted by $90^{\circ}$ with the same magnitude to the four drive electrodes. Under these specific applied drive signals, the three displacement components of the seismic mass are extracted through FEA in response to a frequency sweep. Figure 10 exhibits the displacement magnitude of the central point of the proof-mass as a function of the excitation frequency under $300 \mathrm{mV}$ drive signal amplitude. Indeed, displacements along both $x$ - and $y$-axes are equal and optimum exactly around the first eigen frequency $(1681.5 \mathrm{kHz})$. Displacement along the $z$-axis exists with very low amplitude, so it will not be taken into consideration and we can confirm that the proof-mass undergoes a circular oscillation motion in the $x / y$-plane. As the excitation frequency is in the vicinity of the resonance frequency, it is expected that the structure's vibration will increase, and thus the bias drive voltage will decrease. The choice of the excitation voltage amplitude (Drive mode amplitude) is also essential since it influences the mass tip oscillation magnitude which increases linearly with the applied drive voltage as shown in Figure 11.

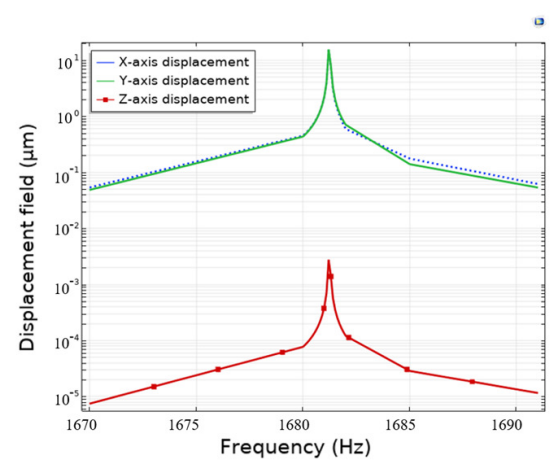

Fig. 10. Proof-mass middle tip displacement in $x$-, $y$ - and $z$-axes vs frequency under drive mode.

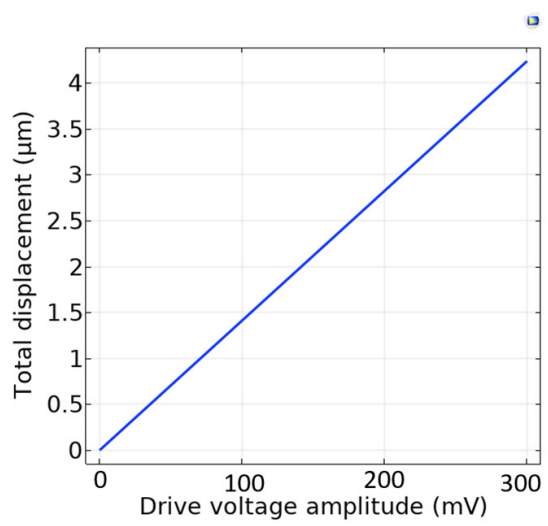

Fig. 11. Proof-mass middle tip displacement in response to varying drive voltage amplitude.

Indeed, the choice of the excitation amplitude must respect the fact that the delivered voltage on sense electrodes due to drive oscillation must be minimal in order be able to visualize acceleration and angular rate effects on sensing voltages. When respecting this assumption, it will be always possible to distinguish motion response, from the oscillation one which is permanently present. It is worth noting that during the oscillation (i.e. in drive mode), the recovered voltage from the ring electrode is insignificant since half of its surface is located in an expansion zone, while the other half is located in a compression zone. Hence, the average stress at the level of the whole connected ring is very weak unlike the S-ring (where the four sense electrodes are located) which is divided into four zones. The velocity components of the proof-mass versus time can be extracted and examined as shown in Figure 12. In fact, as a very much lower displacement of the proof-mass is obtained in the $z$-direction, velocity would also be too low compared to the in-plane components. 


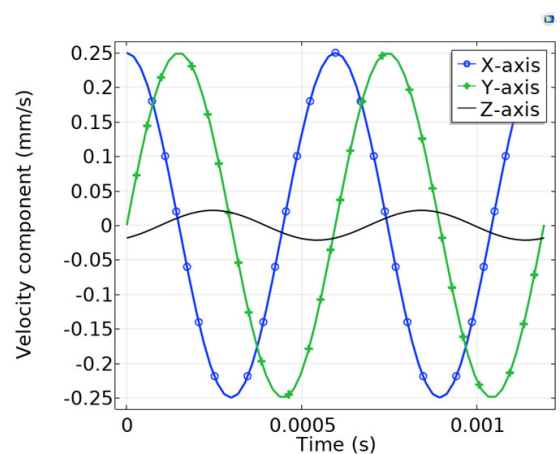

Fig. 12. $x$-, $y$ - and $z$-axis velocity components of the proof-mass oscillation.

\section{B. Linear Acceleration Sensing Evaluation}

Under a lateral acceleration along the $x$ - or $y$-axis, the seismic-mass will tilt in the opposite direction, and thereafter the distributed stress in sense electrodes located on the line application of the acceleration will be positive on one side and negative on the other. Then, a continuous offset voltage, which is proportional to the acceleration undergone by the mass, will be superimposed to the drive voltage generated by the proofmass oscillation as depicted in Figure 13(a). Similarly, in the case of $z$-axis acceleration, the summation of the four sense electrodes together will register an offset voltage which will also be superimposed on the excitation voltage. We note that the offset value of each acceleration output signal under proofmass oscillation is equal to the value corresponding to the pure acceleration effect (Figure 13(b)) which validates the simulation results.

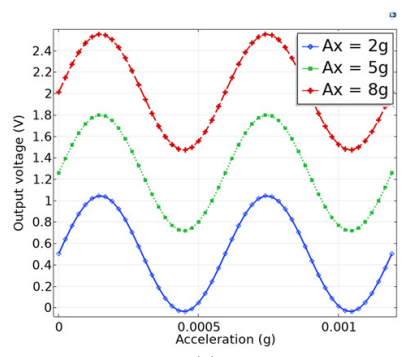

(a)

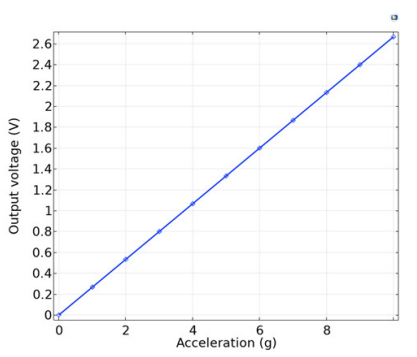

(b)
Fig. 13. $x$-axis acceleration response with the application of (a) drive mode and (b) static mode.

\section{Angular Rate Sensing Evaluation}

When subjected to a rotation motion, the Coriolis force triggers a tilt force and excites the vibration of the second mode, called sense mode. The oscillation amplitude of the generated force will be proportional to the applied angular rate:

$$
F_{C}=2 m_{o s c i}\left(\begin{array}{l}
v_{x} \\
v_{y} \\
v_{z}
\end{array}\right) \wedge\left(\begin{array}{l}
\Omega_{x} \\
\Omega_{y} \\
\Omega_{z}
\end{array}\right)
$$

\section{1) Case 1}

In the presence of angular velocity around the $x$-axis $\Omega_{x}$, two components of Coriolis force are created: one along the $z$ axis and another along the $y$-axis, given by (6) and (7):

$$
\begin{gathered}
F_{y}=2 m_{o s c i} v_{z} \Omega_{x} \\
F_{z}=-2 m_{o s c i} v_{y} \Omega_{x}
\end{gathered}
$$

The numerical values of displacement and velocity components are extracted through FEM simulations,. This allows us to get the magnitude of the produced Coriolis force in $z$ - and $y$-directions as exhibited in Figure 14(a). It is obvious that the $z$-axis component of the Coriolis force dominates since the $x$-direction displacement amplitude is much higher than in the $z$-direction one. Consequently, in response to the $x$-axis angular rotation, the planar oscillation trajectory of the proofmass will be affected by the Coriolis force along the $z$-axis and the effect of this force could be measured from the ring electrode by substituting the shifted new voltage from the initial drive voltage as shown in Figure 14(b). Indeed, the ring voltage keeps its sinusoidal shape, with a higher amplitude which is proportional to the undergone angular rotation. In addition, attention must be paid to the fact that because of the presence of the $y$-axis component of the Coriolis force, the seismic mass will undergo a little additional oscillation stretched in the $y$-direction which can slightly increase the voltage recovered from the Sense electrodes in the $y$-direction. Consequently, the seismic mass will then undergo an elliptical curve oscillation stretched in the $y$-direction instead of a perfect circular trajectory which will increase the amplitude voltage recovered from the electrodes $\mathrm{S}_{\mathrm{y} 1}$ and $\mathrm{S}_{\mathrm{y} 2}$, compared to the one obtained from $\mathrm{S}_{\mathrm{x} 1}$ and $\mathrm{S}_{\mathrm{x} 2}$.

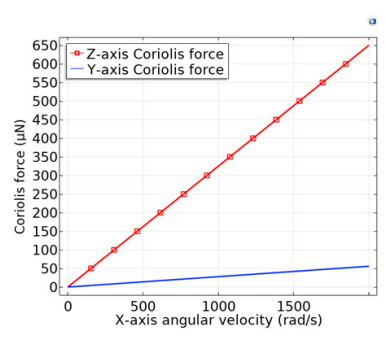

(a)

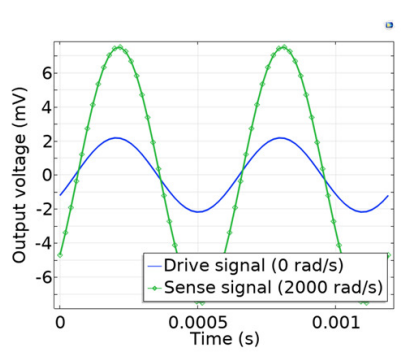

(b)
Fig. 14. (a) Illustration of Coriolis force along the $z$-and $y$-axes in response to $x$-axis angular rate, (b) output voltage from the Ring electrode when subjected to $x$-axis angular rate.

\section{2) Case 2}

In response to the $y$-axis angular velocity input, $\Omega_{y}$, two Coriolis forces are induced. However, only the dominant one is considered, which is along the $z$-axis (same logic as in Case 1). In fact, the velocity oscillator along the $x$-axis is combined with $\Omega_{y}$ to give rise to a dominant Coriolis force along $z$-axis as:

$$
F_{z}=2 m v_{x} \Omega_{y}
$$

Similar to $\Omega_{x}$, the ring electrode will be used to detect the $y$ axis angular rates. However, since $x$ - and $y$-axis proof-mass velocities are $\pi / 2$ phase shifted, then the output signal of $\Omega_{x}$ and $\Omega_{y}$ will also have a $\pi / 2$ phase shift. Hence, a phase detector is proposed to distinguish $\Omega_{x}$ from $\Omega_{y}$.

\section{3) Case 3}

Two Coriolis force components are generated with the same magnitude under the $z$-axis angular rate. This is because 
the $z$-axis angular input is perpendicular to the $x-y$ oscillation plane. Hence, the produced Coriolis force will enclose two compounds:

$$
\begin{gathered}
F_{y}=2 m_{o s c i} v_{x} \Omega_{z} \\
F_{x}=-2 m_{o s c i} v_{y} \Omega_{z}
\end{gathered}
$$

As noticed, the produced forces in $x$ - and $y$-directions are equal but they are phase-shifted due to the phase shift of the initial Drive signals in $x$ - and $y$-directions. Using FEM simulations, under the $z$-axis angular rate, we were able to evaluate the generated Coriolis effect on sense signals. In fact, the diameter of the oscillation circle of the seismic mass will either increase or decrease. Then, the amplitude of the sinusoidal component of all sense electrodes located in the inner ring will either increase or decrease, depending on the direction of the angular rotation. The simulation result of the output voltage is plotted in Figure 15. The output voltage versus time is extracted with the use of the potential difference between electrodes $\mathrm{S}_{\mathrm{x} 1}$ and $\mathrm{S}_{\mathrm{x} 2}$ as well as $\mathrm{S}_{\mathrm{y} 1}$ and $\mathrm{S}_{\mathrm{y} 2}$.

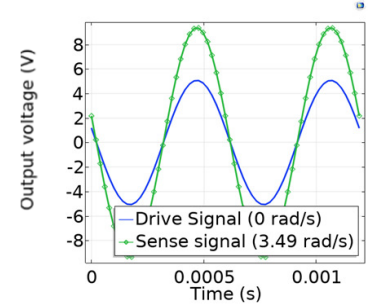

(a)

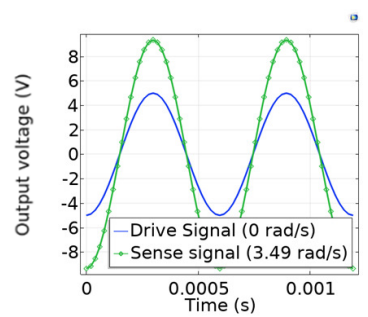

(b)
Fig. 15. Sense voltage in response to the $z$-axis angular rate input: (a) Output voltage $\left(\mathrm{S}_{\mathrm{x} 1}-\mathrm{S}_{\mathrm{x} 2}\right)$ vs. time and (b) output voltage $\left(\mathrm{S}_{\mathrm{y} 1}-\mathrm{S}_{\mathrm{y} 2}\right)$ vs. time.

\section{COMPARISON AND DISCUSSION}

The sensitivity of the studied design is calculated in the three axes for angular velocity and linear acceleration sensing. When the results of the optimized design are compared to the results of the original one, an amelioration rate of sensitivity is found to be up to $165 \%$ for linear acceleration, whereas for angular rate sensing, the lateral sensitivity is ameliorated by about $330 \%$ and is multiplied by a factor of around 10 in the normal axis. Sensitivity values relative to the original and the optimized design are listed in Table II.

TABLE II. PERFORMANCES OF THE ORIGINAL AND THE OPTIMIZED DESIGN IN TERMS OF LINEAR AND ANGULAR SENSITIVITIES

\begin{tabular}{|c|c|c|c|c|}
\hline \multirow{2}{*}{} & \multicolumn{2}{|c|}{ Angular rate sensitivity } & \multicolumn{2}{c|}{ Acceleration sensitivity } \\
\cline { 2 - 5 } & $\boldsymbol{x}$ - and $\boldsymbol{y}$-axis & $\boldsymbol{z}$-axis & $\boldsymbol{x}$ - and $\boldsymbol{y}$-axis & $\boldsymbol{z}$-axis \\
\hline Original design & $0.62 \mu \mathrm{V} / \mathrm{rad} / \mathrm{s}$ & $0.13 \mathrm{~V} / \mathrm{rad} / \mathrm{s}$ & $98 \mathrm{mV} / \mathrm{g}$ & $45 \mathrm{mV} / \mathrm{g}$ \\
\hline Optimized design & $2.65 \mu \mathrm{V} / \mathrm{rad} / \mathrm{s}$ & $1.24 \mathrm{~V} / \mathrm{rad} / \mathrm{s}$ & $260 \mathrm{mV} / \mathrm{g}$ & $60.7 \mathrm{mV} / \mathrm{g}$ \\
\hline
\end{tabular}

The new 6-axis IMU presents very good sensitivity especially in terms of $z$-axis angular velocity and 3-dimentional acceleration detection. This good performance is related to the precise setting of the geometrical design parameters that directly influence the output of the sensor. In fact, taking advantage of the most suitable anchor position, the optimized locations for sensing electrodes, the perfect choice of the operating frequency, and the minute thickness of the detecting element allows designing a high-performance sensor. This method of geometry investigation represents a reliable and efficient way to improve the mechanical and electrical sensor outputs.

\section{CONCLUSION}

Inertial measurement unit sensors are widely used in various applications due to the important information they can provide including acceleration and angular rate data of a movable object. However, because of the use of two separate blocks, one for the accelerometer and one for the gyroscope, there are several limitations bound the IMU development, consisting mainly in size, cost, and energy consumption. Therefore, IMUs are restricted to relative bulk application and thus, despite its importance, the conventional IMU becomes unsuitable to consumer applications unless the size of the devices is reduced. In this paper, we have detailed the design performance of a 6-axes single-mass piezoelectric IMU. The derived FEM model was based on an original design proposed in [25]. A meticulous analysis of geometry investigation was performed including anchor position, optimized locations for sensing and driving electrodes, proof-mass dimensions, and resonance operating frequency. This geometrical analysis highlighted the design optimization and presented a considerable amelioration in terms of linear and angular sensitivity.

\section{REFERENCES}

[1] A. S. Kundu, O. Mazumder, P. K. Lenka, and S. Bhaumik, "Hand Gesture Recognition Based Omnidirectional Wheelchair Control Using IMU and EMG Sensors," Journal of Intelligent \& Robotic Systems, vol. 91, no. 3, pp. 529-541, Sep. 2018, doi: 10.1007/s10846-017-0725-0.

[2] A. Zul Azfar and D. Hazry, "A simple approach on implementing IMU sensor fusion in PID controller for stabilizing quadrotor flight control," in 7th International Colloquium on Signal Processing and its Applications, Penang, Malaysia, Mar. 2011, pp. 28-32, doi: 10.1109/ CSPA.2011.5759837.

[3] D. A. Gura, G. G. Shevchenko, L. F. Kirilchik, D. V. Petrenkov, and T. A. Gura, "Application of inertial measuring unit in air navigation for ALS and DAP," Journal of Fundamental and Applied Sciences, vol. 9, no. 1S, pp. 732-741, Jul. 2017, doi: 10.4314/jfas.v9i1S.

[4] N. Ahmad, R. A. Raja Ghazilla, N. Khairi, and V. Kasi, "Reviews on Various Inertial Measurement Unit (IMU) Sensor Applications," International Journal of Signal Processing Systems, vol. 1, no. 2, pp. 256-262, Jan. 2013, doi: 10.12720/ijsps.1.2.256-262.

[5] F. Aghili and A. Salerno, "Driftless 3-D Attitude Determination and Positioning of Mobile Robots By Integration of IMU With Two RTK GPSs," IEEE/ASME Transactions on Mechatronics, vol. 18, no. 1, pp. 21-31, Feb. 2013, doi: 10.1109/TMECH.2011.2161485.

[6] M. Hutter, C. Gehring, M. Bloesch, M. A. Hoepflinger, C. D. Remy, and R. Siegwart, "StarlETH: A compliant quadrupedal robot for fast, efficient, and versatile locomotion," in 15th International Conference on Climbing and Walking Robot - CLAWAR, Baltimore, USA, Jul. 2012.

[7] G. Fenu and G. Steri, "IMU based post-traumatic rehabilitation assessment," in 3rd International Symposium on Applied Sciences in Biomedical and Communication Technologies, Rome, Italy, Nov. 2010, pp. 1-5, doi: 10.1109/ISABEL.2010.5702813.

[8] P. T. Gibbs and H. Asada, "Wearable Conductive Fiber Sensors for Multi-Axis Human Joint Angle Measurements," Journal of NeuroEngineering and Rehabilitation, vol. 2, no. 1, Mar. 2005, doi: 10.1186/1743-0003-2-7, Art. no. 7.

[9] S.-H. P. Won, F. Golnaraghi, and W. W. Melek, “A Fastening Tool Tracking System Using an IMU and a Position Sensor With Kalman Filters and a Fuzzy Expert System," IEEE Transactions on Industrial 
Electronics, vol. 56, no. 5, pp. 1782-1792, May 2009, doi: 10.1109/ TIE.2008.2010166.

[10] F. Qin, X. Zhan, and G. Du, "Improvement of global navigation satellite system signal acquisition using different grade inertial measurement units for high dynamic applications," IET Radar, Sonar \& Navigation, vol. 8, no. 3, pp. 233-241, Mar. 2014, doi: 10.1049/iet-rsn.2012.0362.

[11] D. Dobriborsci, A. Kapitonov, and N. Nikolaev, "The basics of the identification, localization and navigation for mobile robots," in International Conference on Information and Digital Technologies, Zilina, Slovakia, Jul. 2017, pp. 100-105, doi: 10.1109/DT.2017 .8024279 .

[12] S. Bose, A. K. Gupta, and P. Handel, "On the noise and power performance of a shoe-mounted multi-IMU inertial positioning system," in International Conference on Indoor Positioning and Indoor Navigation, Sapporo, Japan, Sep. 2017, pp. 1-8, doi: 10.1109/ IPIN.2017.8115944.

[13] S. Yean, B. S. Lee, C. K. Yeo, and C. H. Vun, "Algorithm for 3D orientation estimation based on Kalman Filter and Gradient Descent," in IEEE 7th Annual Information Technology, Electronics and Mobile Communication Conference, Oct. 2016, pp. 1-6, doi: 10.1109/ IEMCON.2016.7746263.

[14] A. Beke, A. A. Yuceler, and T. Kumbasar, "A rule based fuzzy gesture recognition system to interact with Sphero 2.0 using a smart phone," in International Artificial Intelligence and Data Processing Symposium, Malatya, Turkey, Sep. 2017, pp. 1-4, doi: 10.1109/IDAP.2017.8090191.

[15] G. Newell and G. Vejarano, "Human-motion based transmission power control in wireless body area networks," in IEEE 3rd World Forum on Internet of Things, Reston, VA, USA, Dec. 2016, pp. 277-282, doi: 10.1109/WF-IoT.2016.7845404.

[16] D. K. Shaeffer, "MEMS inertial sensors: A tutorial overview," IEEE Communications Magazine, vol. 51, no. 4, pp. 100-109, Apr. 2013, doi: 10.1109/MCOM.2013.6495768.

[17] S. A. Alqarni, A. M. Obeid, M. S. BenSaleh, and S. M. Qasim, "A fully integrated CMOS interface ASIC for two-axis piezoelectric angular rate MEMS inertial sensors," in IEEE SENSORS, Busan, South Korea, Nov. 2015, pp. 1-2, doi: 10.1109/ICSENS.2015.7370382.

[18] K. Hari, S. K. Verma, I. R. Praveen Krishna, and V. Seena, "Out-ofplane dual flexure MEMS piezoresistive accelerometer with low cross axis sensitivity," Microsystem Technologies, vol. 24, no. 5, pp. 24372444, May 2018, doi: 10.1007/s00542-017-3679-z.

[19] Y. V. Filatov, A. M. Boronakhin, V. B. Dao, V. C. Le, and L. N. Podgornaya, "Studying the static errors of MEMS accelerometer triad in quasiharmonic oscillation mode," Gyroscopy and Navigation, vol. 8, no. 2, pp. 121-128, Apr. 2017, doi: 10.1134/S2075108717020055.

[20] L. Meyer, A. Buhmann, R. Eid, and J. G. Korvink, "Root Cause Analysis of Zero-Rate Output Sources in an MEMS Gyroscope," IEEE Sensors Journal, vol. 17, no. 4, pp. 959-966, Feb. 2017, doi: 10.1109/ JSEN.2016.2636563.

[21] S. Dellea, P. Rey, and G. Langfelder, "MEMS Gyroscopes Based on Piezoresistive NEMS Detection of Drive and Sense Motion," Journal of Microelectromechanical Systems, vol. 26, no. 6, pp. 1389-1399, Dec. 2017, doi: 10.1109/JMEMS.2017.2749121.

[22] C. Acar, "Micromachined monolithic 6-axis inertial sensor," US 9,278,846 B2, Mar. 2016.

[23] R. Amarasinghe, D. V. Dao, T. Toriyama, and S. Sugiyama, "Development of miniaturized 6-axis accelerometer utilizing piezoresistive sensing elements," Sensors and Actuators A: Physical, vol. 134, no. 2, pp. 310-320, Mar. 2007, doi: 10.1016/j.sna.2006.05.044.

[24] J. Liu, M. Li, L. Qin, and J. Liu, "Principle Research on a Single Mass Piezoelectric Six-Degrees-of-Freedom Accelerometer," Sensors, vol. 13, no. 8, pp. 10844-10855, Aug. 2013, doi: 10.3390/s130810844.

[25] K. Okada, T. Kakutani, H. Itano, Y. Matsu, and S. Sugiyama, "Development of 6-axis Motion Sensors Using Piezoelectric Elements," in 21st Sensor Symposium, Kyoto, Japan, Oct. 2004, pp. 385-390.

[26] K. Okada, T. Kakutani, and Y. Matsu, "Development of the 3-axis Angular Velocity Sensor Using a Piezoelectric Element," IEEJ Transactions on Sensors and Micromachines, vol. 125, no. 6, pp. 272277, 2005, doi: 10.1541/ieejsmas. 125.272.
[27] C. Liu, Foundations of MEMS, 2nd Edition. London, UK: Pearson, 2012.

[28] J. M. Calderon-Moreno, "Stress induced domain switching of PZT in compression tests," Materials Science and Engineering: A, vol. 315, no. 1, pp. 227-230, Sep. 2001, doi: 10.1016/S0921-5093(01)01154-6.

[29] M. D. Nguyen, M. Dekkers, H. N. Vu, and G. Rijnders, "Film-thickness and composition dependence of epitaxial thin-film PZT-based masssensors," Sensors and Actuators A: Physical, vol. 199, pp. 98-105, Sep. 2013, doi: 10.1016/j.sna.2013.05.004.

[30] S. Bhuvana, S. H. Prathiksha, V. T. Sindhu, and H. Vasudha, "Design and Analysis of Piezoelectric Cantilever Based Vibration Sensor," in International Conference on System, Computation, Automation and Networking, Pondicherry, India, Jul. 2018. 